\title{
Average volume-assured pressure support
}

\author{
Abdurahim Aloud MD
}

\begin{abstract}
Average volume-assured pressure support (AVAPS) is a relatively new mode of noninvasive positive pressure ventilation (NiPPV); only a few studies have been done to compare its effectiveness and safety to bilevel positive airway pressure (BiPAP) in chronic respiratory failure secondary to obesity hypoventilation syndrome, obstructive sleep apnea, chronic obstructive pulmonary disease, and neuromuscular disorders with respiratory muscle weakness. Only six studies were found in PubMed, and these studies had many limitations, especially small sample sizes. This review provides detailed summaries of these studies. These devices require more investigation.
\end{abstract}

Keywords: non-invasive ventilation, pressure support, average volume-assured pressure support, obesity-hypoventilation syndrome, COPD, hypercapnia

\section{BACKGROUND}

Average volume-assured pressure support (AVAPS) and intelligent VAPS (iVAPS) are forms of non-invasive positive pressure ventilation (NiPPV) that adjust the pressure support (PS) to maintain a target average ventilation over several breaths. Both AVAPS and iVAPS adjust PS and the respiratory rate to reach a defined target with the goal of stabilizing the $\mathrm{PaCO}_{2}$, which relates directly to alveolar ventilation. ${ }^{1}$

With a target tidal volume (e.g., AVAPS), if there is a variance in the respiratory mechanics, especially compliance, which in turn can change the tidal volume, there can be fluctuations in the alveolar ventilation and thus $\mathrm{PaCO}_{2}$. By targeting the estimated alveolar ventilation (minute ventilation-estimated dead space ventilation; e.g., iVAPS), variations in respiratory mechanics should not affect alveolar ventilation or $\mathrm{PaCO}_{2}$ as long as the estimated dead space

Corresponding author: Abdurahim Aloud

Contact Information: Abdurahim.aloud@ttuhsc.edu DOI: $10.12746 /$ swrccc.v6i22.438 equals physiologic dead space. The device estimates the anatomic dead space using height. However, patients with lung diseases, such as emphysema, have increased physiologic dead space that would be underestimated using their height, and thus their alveolar ventilation might be much lower than the estimated alveolar ventilation. Thus, emphysema patients may require a higher target alveolar ventilation to achieve adequate alveolar ventilation. The "height" can be entered artificially high in emphysema patients to provide a calculated dead space closer to their physiologic dead space, and iVAPS will provide the desired alveolar ventilation.

Average volume-assured pressure support targets an average tidal volume over several breaths. Typically, the target tidal volume is set based on $6-10 \mathrm{~mL} / \mathrm{kg}$ ideal body weight. It calculates the average PS provided to the patient over the prior 2 minutes to achieve a particular tidal volume. If average recent ventilation is less than the target volume, inspiratory positive airway pressure (IPAP) for the next breath is increased. Pressure support will change at a rate of $2 \mathrm{~cm} \mathrm{H}_{2} \mathrm{O}$ /minute if there is unstable breathing and $1 \mathrm{~cm} \mathrm{H}_{2} \mathrm{O}$ /minute if there is stable breathing. The AVAPS-AE model can set 
a maximum rate of pressure change from $1 \mathrm{~cm} \mathrm{H}_{2} \mathrm{O}$ / minute to $5 \mathrm{~cm} \mathrm{H}_{2} \mathrm{O}$ /minute. Expiratory positive airway pressure (EPAP) is fixed with AVAPS, but AVAPS-AE adjusts EPAP as well. Average volume-assured pressure support uses either a fixed rate or auto backup rate set at 2 breaths per minute lower than the rate of the last six spontaneous breaths.

This mode of ventilation can help patients with respiratory insufficiency due to neuromuscular and restrictive disorders in which the respiratory effort varies during sleep, ${ }^{2}$ patients who need NiPPV during the day, patients with COPD at risk for hypoventilation, and patients with obesity hypoventilation (OHS) who may need compensation based on position and/or sleep stage changes. Because many patients have much worse hypoventilation in REM, BiPAP with fixed PS may provide too much pressure in NREM, which may lead to intolerance or complex sleep apnea and may not provide enough PS in REM to control $\mathrm{PaCO}_{2}$ levels.

The theoretical benefits of VAPS over BiPAP include maintaining volumes in the setting of altered patient effort based on sleep stage or altered lung mechanics related to position. Less PS while awake may increase comfort and aid sleep onset, reduce the risk of barotrauma, and provide lower pressures most of the time. A randomized trial of iVAPS vs BiPAP found that iVAPS delivered a lower mean PS for oxygenation and transcutaneous $\mathrm{PaCO}_{2}$ levels and promoted better adherence than $\mathrm{BiPAP}^{3}$

This literature review provides a detailed summary of studies with AVAPS in patients with chronic respiratory failure, OHS, and COPD with acute hypercapneic exacerbations (also see Table).

\section{Study \# 1-Sleep and non-invasive ventilation in patients with chronic respiratory insufficiency ${ }^{2}$}

\section{Study goals}

- This study tests the hypothesis thatAVAPS and the lateral decubitus position in patients with chronic respiratory insufficiency are associated with better sleep efficiency than NIV- PS and the supine position. The secondary aim of the study was to assess the effect of the mode of ventilation, body position, and sleep-wakefulness state on minute ventilation in the same patients.

\section{Methods}

- A single blind, randomized, cross-over, prospective trial studied the effects of NIV-PS vs AVAPS on sleep efficiency in twenty-eight patients with chronic respiratory insufficiency (OHS with or without OSA, COPD, neuromuscular disease).

- Thirty-nine patients with the diagnosis of chronic respiratory insufficiency who were currently receiving home ventilation (NIV-PS) for at least two months and were adherent to this therapy (4 hours per night) were recruited. Exclusion criteria included hemodynamic instability, a history of CHF or Cheyne Stokes respiration, inability to clear secretions, and acute sinusitis, otitis media, facial trauma, or other anatomical abnormalities interfering with mask fit. The study was conducted in a hospital ward.

- Each subject underwent three consecutive overnight sleep studies: 1) Conventional NIV-PS set at the patients' prescription settings to validate the prescription pressures and to serve as an acclimatization night, 2) AVAPS or NIV therapy assigned randomly, and 3) cross- over to alternate therapy mode. Patients underwent randomization only if their prescription pressure did not change significantly $\left(>5 \mathrm{~cm} \mathrm{H}_{2} \mathrm{O}\right.$ ) during the first sleep study. Eleven patients were excluded (central apneas, $\mathrm{n}=3$; pressure change $>5 \mathrm{~cm} \mathrm{H}_{2} \mathrm{O}, \mathrm{n}=6$; refractory hypoxemia, $n=1$; inadequate sleep, $n=1$ ).

\section{Results}

- Twenty-eight patients had adequate pressure settings and received BiPAP or AVAPS on separate nights. The IPAP level during BiPAP remained fixed at $17 \pm 4 \mathrm{~cm} \mathrm{H} \mathrm{H}_{2} \mathrm{O}$. During AVAPS therapy, the set IPAPmin and IPAPmax were $13 \pm 4$ and $26 \pm 3 \mathrm{~cm} \mathrm{H}_{2} \mathrm{O}$, respectively. During AVAPS therapy, the mean IPAP pressure was $18 \pm 5 \mathrm{~cm} \mathrm{H}_{2} \mathrm{O}$ which tended to be higher than IPAP during BiPAP (17 $\pm 4 \mathrm{~cm} \mathrm{H} 2 \mathrm{O}$; $\mathrm{P}=0.08$; paired $t$ test), and the IPAP during AVAPS therapy fluctuated between a low of $14 \pm 6 \mathrm{~cm} \mathrm{H}_{2} \mathrm{O}$ and a high of $21 \pm 5 \mathrm{~cm} \mathrm{H}_{2} \mathrm{O}$. 


\begin{tabular}{|c|c|c|c|}
\hline & 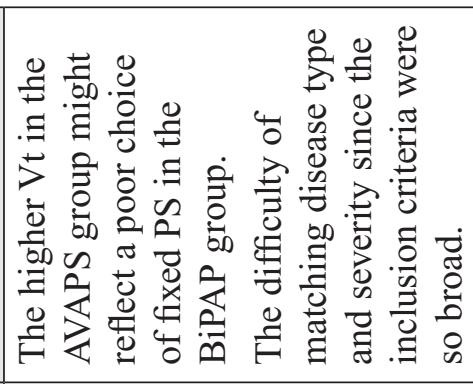 & 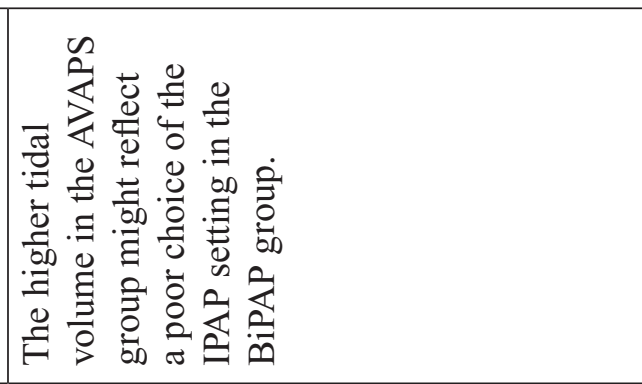 & \\
\hline & 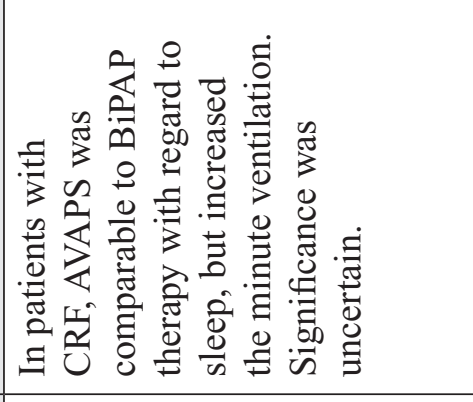 & 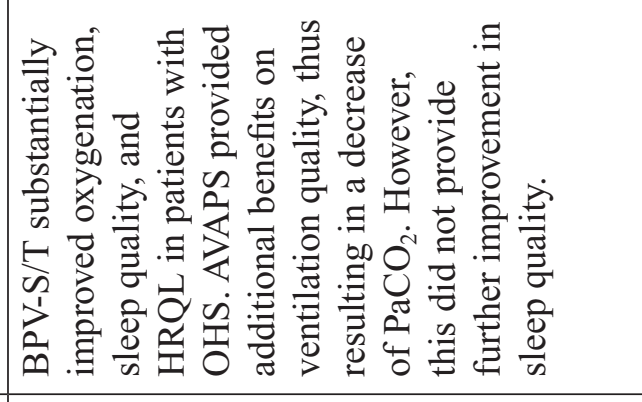 & 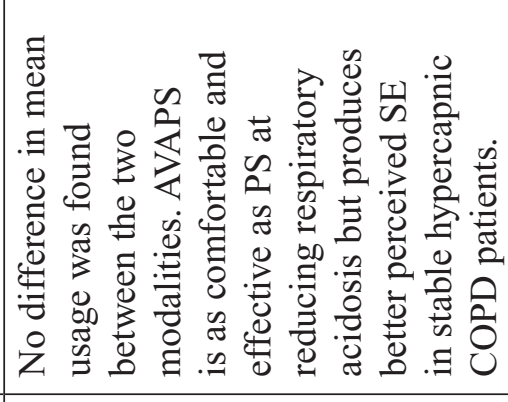 \\
\hline & 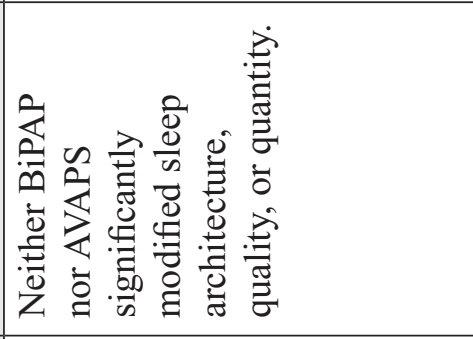 & 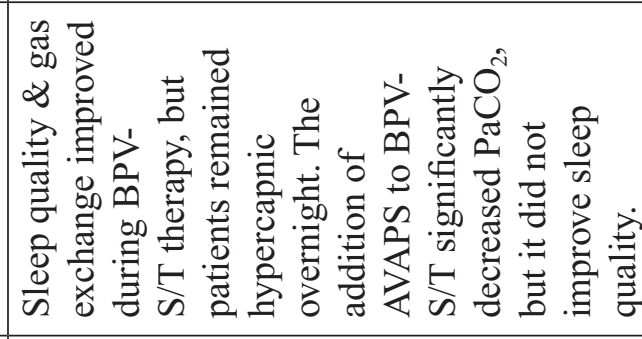 & 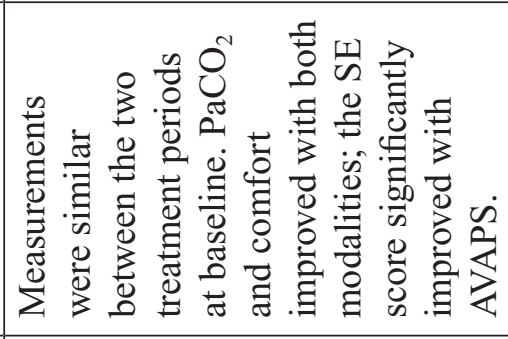 \\
\hline 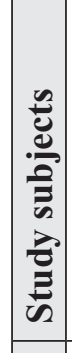 & 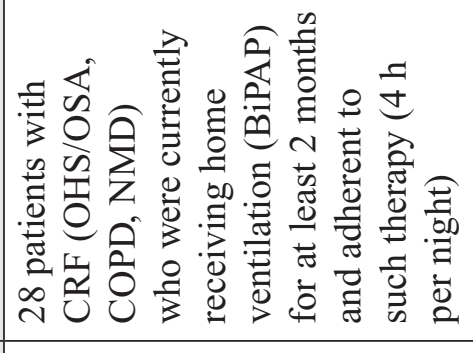 & 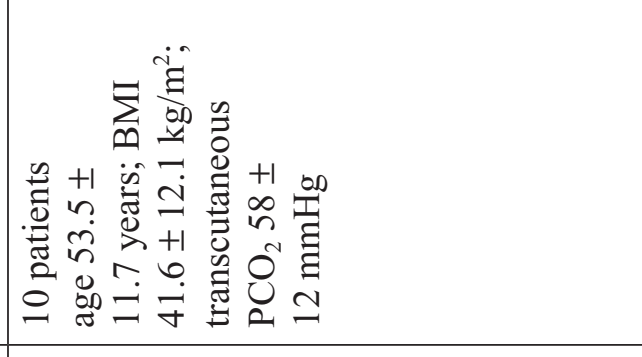 & 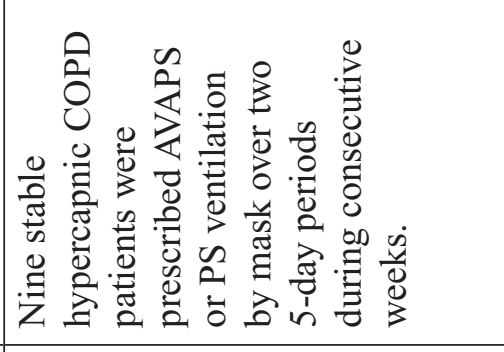 \\
\hline & 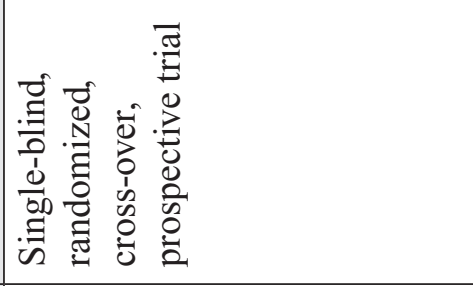 & 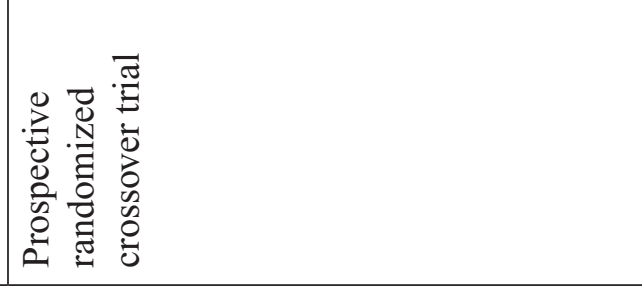 & 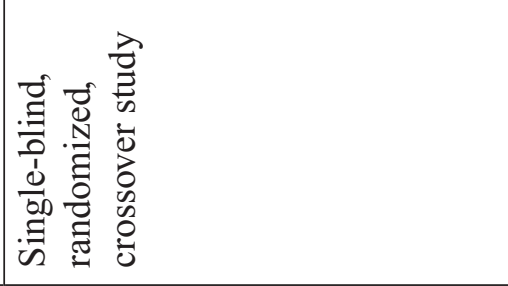 \\
\hline 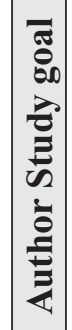 & 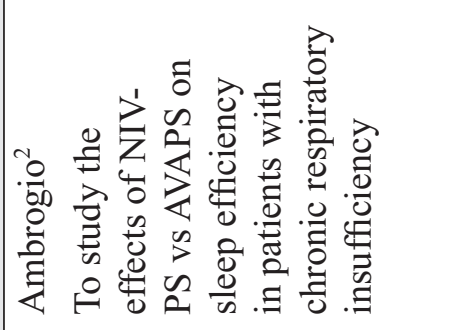 & 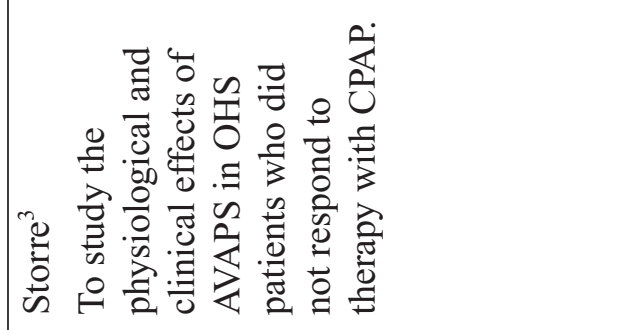 & 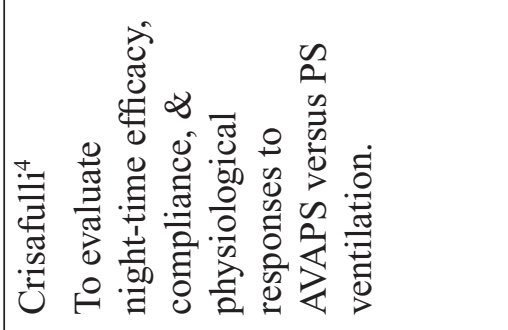 \\
\hline
\end{tabular}




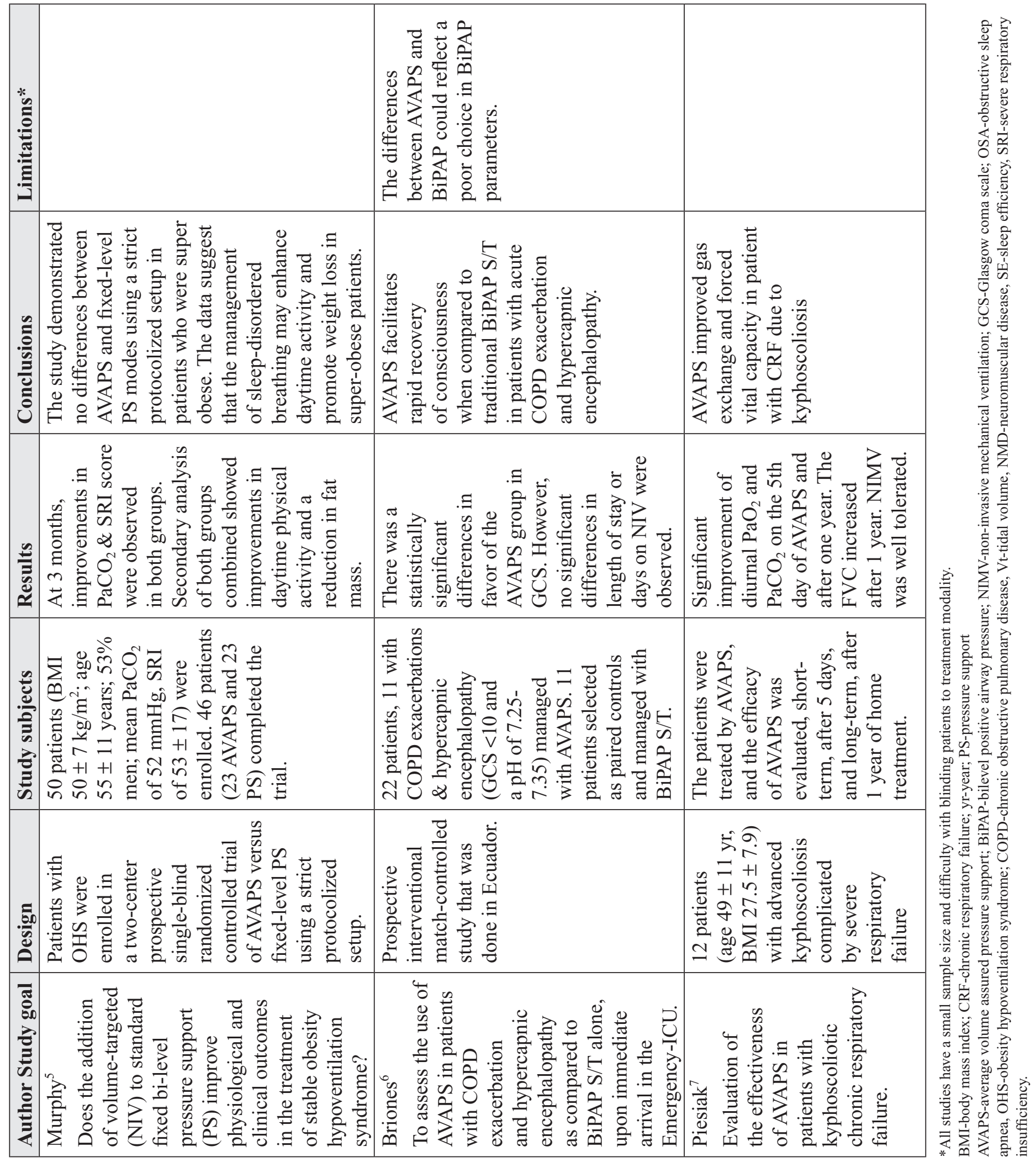


- Sleep architecture was similar in both modes. However, the supine body position was associated with decreased sleep efficiency when compared to the lateral decubitus position $(77.9 \pm 22.9 \%$ and $85.2 \pm 10.5 \% ; P=0.04)$. The apnea-hypopnea index was greater during the supine position (median 6.3) than during lateral position (median 0.6).

- Minute ventilation decreased progressively from wakefulness through various stages of sleep (stage 1 , stage 2, and REM, $P=0.0001$ ) during BiPAP therapy in the supine position. During BiPAP therapy in the lateral decubitus position, minute ventilation decreased progressively from wakefulness through various stages of sleep (stage 1 , stage 2 , and REM, $P=0.018$ ). During AVAPS in the supine position, the minute ventilation did not change from wakefulness through the various stages of sleep.

\section{Conclusions}

- In patients with chronic respiratory insufficiency, the supine position was associated with worse sleep efficiency than the lateral decubitus position. Average volume-assured pressure support was comparable to BiPAP therapy with regards to sleep, but a statistically greater minute ventilation during AVAPS than BiPAP was observed. Minute ventilation was determined by sleep-wakefulness state, body mass index (BMI), and mode of therapy, but not by body position. Changes in minute ventilation during BiPAP therapy were independent of effects of body position and BMI, were due primarily to decreases in tidal volume rather than in respiratory rate, and were less likely during AVAPS.

\section{Study \# 2-Average volume-assured pressure support in obesity hypoventilation ${ }^{3}$}

\section{Study Goals}

- This small prospective randomized crossover trial studied the physiological and clinical effects of AVAPS in 10 OHS patients who did not respond to CPAP. The effects of bilevel pressure ventilation with the spontaneous /timed (BPV with S/T) mode with and without AVAPS over 6 weeks on ventilation pattern, gas exchange, sleep quality, and health related quality of life (HRQL) assessed by severe respiratory insufficiency questionnaire (SRI) were prospectively investigated in a randomized crossover trial.

\section{Methods}

- Inclusion criteria: Clinically stable OHS patients with a body mass index of $30 \mathrm{~kg} / \mathrm{m}^{2}$ and daytime hypercapnia $\left(\mathrm{PaCO}_{2}>45 \mathrm{~mm} \mathrm{Hg}\right)$ who had failed to respond to CPAP therapy, had no other cause for their chronic respiratory failure, and were naive to any ventilatory treatment. Exclusion criteria: Patients who had evidence of acute respiratory failure (patients with worsening symptoms during the last 2 weeks, a breathing frequency of 30 breaths/ minute, a $\mathrm{pH}<7.35$, or signs of respiratory infections). Patients who had been intubated during the last 3 months or had received any other form of ventilatory support prior to hospital admission were also excluded from the study. A CPAP responder was defined as a patient who achieved a transcutaneous $\mathrm{PaCO}_{2}$ level of $<45 \mathrm{~mm} \mathrm{Hg}$ and a respiratory disturbance index (RDI) score of $<10$ events per hour.

- CPAP nonresponders were discharged from the hospital to home with therapy with BPV-S/T with or without AVAPS following randomization and were readmitted to the hospital after 6 weeks of home therapy. Baseline measurements were repeated, and patients were switched to the alternate mode of BPV-S/T. Measurements were again performed after another period of 6 weeks home therapy following hospital readmission.

- Measurements: pulmonary function tests, daytime blood gas at rest, HRQL measured using the RSI questionnaire, polysomnography, and $\mathrm{PtcCO}_{2}$ measured during the night at baseline, and during therapy with CPAP, BPV-S/T, and BPV-S/TAVAPS. Measurements of ventilation were made during therapy with both modes of BPV-S/T using a pneumotachograph to measure volumes, pressures, and respiratory rate.

Results

- Ten patients (mean \pm SD; age $53.5 \pm 11.7$ years; BMI $41.6 \pm 12.1 \mathrm{~kg} / \mathrm{m}^{2}$; FEV1/FVC ratio, $79.4 \pm 6.5 \%$ 
predicted; transcutaneous $\mathrm{PCO}_{2} 58 \pm 12 \mathrm{~mm} \mathrm{Hg}$; IPAP for BPV $17 \pm 2 \mathrm{~cm} \mathrm{H}_{2} \mathrm{O}$; tidal volume for AVAPS $7 \mathrm{ml} / \mathrm{kg}$ [five patients] and $10 \mathrm{ml} / \mathrm{kg}$ [five patients]) completed the study. $\mathrm{PtcCO}_{2}$ non-significantly decreased during nocturnal BPV-S/T by -5.6 $\pm 11.8 \mathrm{~mm} \mathrm{Hg}(95 \%$ confidence interval [Cl], -14.7 to $3.4 \mathrm{~mm} \mathrm{Hg} ; \mathrm{p}=0.188$ ), but significantly decreased during AVAPS by $-12.6 \pm 12.2 \mathrm{~mm} \mathrm{Hg}(95 \% \mathrm{Cl}$, -22.0 to-3.2mmHg; $p=0.015)$.Pneumotachographic measurements revealed a higher individual variance of peak inspiratory pressure $(p<0.001)$ and a trend for lower leak volumes and for higher tidal volumes during AVAPS. Sleep quality and oxygen saturation also comparably improved using both BPV-S/T and AVAPS.

- Sleep quality and gas exchange substantially improved during nocturnal BPV-S/T therapy compared to baseline, but patients remained hypercapnic overnight even after 6 weeks of BPV-S/T therapy. The addition of AVAPS to BPV-S/T therapy resulted in a significant decrease of $\mathrm{PaCO}_{2}$ and normalized $\mathrm{PaCO}_{2}$ during sleep. However, this reduction in $\mathrm{PaCO}_{2}$ did not lead to further improvements in sleep quality compared to standard BPV-S/T therapy. Daytime $\mathrm{PaCO}_{2}$ and bicarbonate levels significantly improved following AVAPS therapy only.

\section{Conclusions}

- The BPV-S/T mode substantially improved oxygenation, sleep quality, and HRQL in patients with OHS. Average volume-assured pressure support provided additional benefits on ventilation quality and resulted in a bigger decrease in $\mathrm{PaCO}_{2}$. However, this did not provide further clinical benefits in sleep quality and HRQL.

\section{Study \# 3-Subjective sleep quality during AVAPS ventilation in patients with hypercapnic COPD: a physiological pilot study 4}

\section{Study Goals}

- This single-blind (ventilator modalities were blinded to the patients), randomized, crossover study evaluated night-time efficacy, compliance, and physiological responses to AVAPS versus PS ventilation.

\section{Methods}

- Average volume-assured pressure support or PS ventilation was delivered to nine stable hypercapnic COPD patients by a mask over two 5-day periods during consecutive weeks. The Synchrony ventilator was used to deliver mask ventilation as follows: $8 \mathrm{ml} / \mathrm{kg}$ of ideal body weight (as the targeted inspiratory tidal volume) with IPAP ranging from EPAP up to $30 \mathrm{~cm} \mathrm{H}_{2} \mathrm{O}$ for AVAPS, and the patient's highest tolerated IPAP level for PS. Expiratory positive airway pressure was set at the minimum level for both modalities and oxygen was added at a fixed inspiratory fraction to maintain $\mathrm{SaO}_{2}$ above $90 \%$.

- Inclusion criteria: stable chronic hypercapnia at rest without respiratory acidosis $\mathrm{PaCO}_{2}$ $>45 \mathrm{mmHg}, \mathrm{pH}>7.35$, severe COPD (stage III or IV) according to GOLD classification, naive to NPPV, and apnea/hypopnea index $(\mathrm{AHI})<10$ episodes/hour as assessed by preliminary polysomnography. Exclusion criteria: patients with unstable hemodynamic and respiratory conditions (e.g., recent acute heart failure, respiratory exacerbation or infection with worsening symptoms during the preceding four weeks, and/or changes in any domiciliary therapy in the last week), malignancies, or an inability to cooperate.

- Measurements: Demographic and anthropometric variables (age, sex, BMI) and lung function parameters (FEV $\left.1, F V C, F E V_{1} / F V C, R V, T L C\right)$, voluntary respiratory muscle strength values (MIP and MEP). Compliance to ventilation, as measured by mean usage (hours/night), arterial blood gases, comfort, and perceived sleep efficiency (SE by questionnaire) were measured at baseline (TO) and after three (T1) and five (T2) nights over the two periods.

\section{Results}

- Measurements were similar between the two treatment periods at baseline. $\mathrm{PaCO}_{2}$ and comfort improved with both modalities; the SE score significantly improved at T2 with AVAPS (from 
$5.1 \pm 2.0$ to $4.1 \pm 2.2, \mathrm{P}=0.001$ ) but not with $\mathrm{PS}$ (from $5.1 \pm 1.7$ to $4.7 \pm 1.3, P=0.219$ ).

\section{Conclusions}

- No difference in mean usage was found between the two modalities. Mask AVAPS was as comfortable and effective as PS at reducing respiratory acidosis but produced better perceived sleep efficiency in stable hypercapnic COPD patients.

\section{Study \# 4-Volume targeted versus pressure support non-invasive ventilation in patients with "super" obesity and chronic respiratory failure: a randomized controlled trial ${ }^{5}$}

\section{Study Goals}

- A prospective single-blind randomized controlled trial of AVAPS versus fixed-level PS using a strict protocol done in two centers investigated the effects of the addition of AVAPS to standard BiPAP on the physiological and clinical outcomes in the treatment of stable OHS.

\section{Methods}

- Inclusion criteria: $\mathrm{BMI}>40 \mathrm{~kg} / \mathrm{m}^{2}$; daytime stable respiratory failure with $\mathrm{PaCO}_{2}>45 \mathrm{mmHg}$ and $\mathrm{pH}$ >7.35; absence of another identifiable cause of hypoventilation; $\mathrm{FEV}_{1} / \mathrm{FVC}>0.70$; and $\mathrm{FVC}<70 \%$ predicted. Exclusion criterion: an inability to provide written informed consent.

- 50 patients (BMI $50 \pm 7$; age $55 \pm 11$ years; $53 \%$ men, with a mean $\mathrm{PaCO}_{2}$ of $51.8 \mathrm{mmHg}$ and SRI of $53 \pm 17$ ) were enrolled in the study. Patients were randomly allocated to either fixed bi-level PS or AVAPS mode. Both modes were delivered by a BiPAP synchrony device. Pre-randomization minimization was performed to avoid allocation bias; variables were BMI $(40-50,50-60$, and $>60)$, neck circumference $(<45 \mathrm{~cm}$ and $>45 \mathrm{~cm})$, gender, and clinical presentation (acute or elective).

- Measurements: Baseline spirometry, arterial blood gases, anthropometrics including body composition measurements, HRQL assessed by severe respiratory insufficiency questionnaire (SRI), and self-reported sleep comfort. Following randomization, patients underwent respiratory polygraphy, including oximetry and measurement of tcPCO ${ }_{2}$, and supplemental oxygen was provided to patients who met the criteria for daytime hypoxemia. Once established on NIV, patients were discharged and followed up at 3 months.

- The primary outcome was change in daytime arterial $\mathrm{PaCO}_{2}$ at 3 months. Body composition, physical activity, and HRQL (severe respiratory insufficiency questionnaire, SRI) were secondary outcome measures.

Results

- 46 patients (23 AVAPS and 23 PS) completed the trial. At 3 months, improvements in $\mathrm{PaCO}_{2}$ occurred in both groups (AVAPS $\Delta 4.5 \mathrm{mmHg}, 95 \% \mathrm{Cl} 1.5$ to $8.25, \mathrm{p}<0.01$ vs PS $\Delta 4.5 \mathrm{mmHg}, 95 \% \mathrm{Cl} 0.75$ to $8.25, p=0.02$ ) but no between group difference $(\Delta-0.7 .5 \mathrm{mmHg}, 95 \% \mathrm{Cl} 5.25$ to $4, \mathrm{p}=0.87)$. The SRI improved in both groups (AVAPS $\Delta 11,95 \%$ Cl 6 to $17, \mathrm{p}<0.001$ vs PS $\Delta 7,95 \% \mathrm{Cl} 1$ to 12 , $\mathrm{p}=0.02$; between groups $\Delta 5,95 \% \mathrm{Cl}-3$ to 12 , $p=0.21$ ). Secondary analysis of both groups combined showed improvements in daytime physical activity that correlated with reductions in fat mass.

\section{Conclusions}

- The study demonstrated no differences between automated AVAPS mode and fixed-level PS mode using a strict protocol setup in patients who were "super" obese. The data suggest that the management of sleep-disordered breathing may enhance daytime activity and promote weight loss in "super" obese patients.

\section{Study \# 5-Noninvasive mechanical ventilation with AVAPS in patients with COPD and hypercapnic encephalopathy ${ }^{6}$}

\section{Study Goals}

- This study assessed the use of AVAPS in patients with COPD exacerbation and hypercapnic encephalopathy compared to BiPAP S/T 
alone upon immediate arrival to the Emergency Department or the ICU.

\section{Methods}

- This was a prospective interventional match-controlled study with 22 patients. Eleven patients with COPD exacerbations and hypercapnic encephalopathy with a Glasgow Coma Scale (GCS) $<10$ and a $\mathrm{pH}$ of 7.25-7.35 were assigned to receive AVAPS. Eleven patients were selected as paired controls for the initial group by physicians who were unfamiliar with the study, and these patients were managed with BiPAP S/T. Arterial blood gases, GCS, vital signs, and ventilatory parameters were then measured and compared between the two groups.

\section{Results}

- There was a statistically significant difference in favor of the AVAPS group in GCS $(P=.00001)$, $\mathrm{PaCO}_{2}(\mathrm{P}=.03)$ and maximum IPAP $(\mathrm{P}=.005)$. However, no significant differences in length of stay or days on NIV were observed.

\section{Conclusions}

- Average volume-assured pressure support facilitates rapid recovery of consciousness when compared to traditional BiPAP $S / T$ in patients with acute COPD exacerbation and hypercapnic encephalopathy. Average volume-assured pressure support is also a safe strategy of noninvasive ventilatory treatment in patients with exacerbations of COPD and hypercapnic encephalopathy (GCS $<10$ ), with the caveat that these patients should be treated in units with ample experience and under close surveillance.

\section{Study \# 6-Efficacy of AVAPS in patients with chronic respiratory failure due to kyphoscoliosis ${ }^{7}$}

\section{Study Goals}

- This study evaluated the effectiveness of AVAPS in patients with kyphoscoliosis and chronic respiratory failure.

\section{Methods}

- The study included 12 patients (mean age $49 \pm 11$ years and body mass index $27.5 \pm 7.9 \mathrm{~kg} / \mathrm{m}^{2}$ ) with advanced kyphoscoliosis complicated by severe respiratory failure $\left(\mathrm{PaCO}_{2} 650.1 \pm 2.6 \mathrm{mmHg}\right.$, $\mathrm{SaO}_{2} 81.7 \pm 3.1 \%, \mathrm{PaCO}_{2} 71.3 \pm 8.1 \mathrm{mmHg}$ ) treated by AVAPS. The short-term after 5 days and long-term after 1 year of home treatment efficacy of AVAPS was evaluated.

\section{Results}

- The study found a significant improvement of diurnal $\mathrm{PaO}_{2}$ and $\mathrm{PaCO}_{2}$ on the 5th day of AVAPS (an increase of $10.4 \pm 2.3 \mathrm{mmHg}$ and a decrease of $13.5 \pm 6.0 \mathrm{mmHg}$, respectively; $p<0.05$ ) and after one year AVAPS (an increase of $15.5 \pm 3.5 \mathrm{mmHg}$ and a decrease of $20.1 \pm 6.5 \mathrm{mmHg}$, respectively; $p<0.05)$. There was a significant increase of mean blood oxygen saturation during sleep on the 5th day $(86.2 \pm 3.2 \%)$ and after 1 year of treatment $(89.4 \pm 2.1 \%)$ compared with the baseline level $(83.2 \pm 3.2 \%)$. The forced vital capacity also increased after 1 year $(1,024 \pm 258 \mathrm{ml}$ vs. the baseline $908 \pm 267 \mathrm{ml}$; $p<0.05)$. Average volume-assured pressure support was well tolerated, and no patient discontinued the treatment during the observation period.

\section{Conclusions}

- Average volume-assured pressure support decreased the $\mathrm{PaCO}_{2}$ and increased the $\mathrm{PaO}_{2}$ and FVC in patients with kyphoscoliosis and chronic respiratory failure.

\section{SUMMARY}

These six studies included 127 patients who were managed with either AVAPS or BiPAP or both modes of non-invasive ventilation (See Table for summary of study results).

For Outpatient management: There was no difference between the two modalities in improving sleeping efficiency and HRQL in patients with chronic 
respiratory insufficiency due to OHS with or without OSA, COPD, and neuromuscular disease. There was no difference in mean usage between the two modalities. Mask AVAPS was as comfortable and as effective as PS at reducing respiratory acidosis in stable hypercapnic COPD patients. There were no differences between using AVAPS and fixed-level PS mode in patients who were "super" obese. AVAPS provided additional benefits on ventilation quality, thus resulting in a more efficient decrease of $\mathrm{PaCO}_{2}$. However, this did not provide further clinical benefits in sleep quality and $\mathrm{HRQL}$, and this result has uncertain significance.

For Inpatient management: In patients with acute COPD exacerbation and hypercapnic encephalopathy, AVAPS facilitates rapid improvement in the level of consciousness when compared to traditional BiPAP S/T. However, no significant differences in length of stay or days on NIV were observed. AVAPS is a safe strategy of noninvasive ventilatory treatment in patients with exacerbations of COPD and hypercapnic encephalopathy $(\mathrm{GCS}<10)$. These studies do not identify a clinical scenario in which AVAPS has a definite advantage when compared to bi-level pressure support. Patients managed with non-invasive mechanical ventilation need careful monitoring with defined clinical goals to improve outcomes. The exact mode may be less important.

Criticisms common to all these studies include the small sample size and the difficulty with blinding patients to the treatment modality. The higher tidal volumes in the AVAPS groups may not reflect an advantage of AVAPS but rather a poor choice of fixed pressure support in the BiPAP group.

\section{ReCOMMENDATIONS}

1. For Outpatient management: AVAPS is as safe and as effective as BiPAP but is not superior to it in the management of outpatients with chronic respiratory insufficiency due to OHS with or without OSA, COPD, and neuromuscular disease.
2. For Inpatient management: AVAPS might be preferred over BiPAP for patients with acute COPD exacerbation and hypercapnic encephalopathy as it facilitates more rapid improvement in the level of consciousness.

Article citation: Aloud A. Average volume-assured pressure support. The Southwest respiratory and Critical care chronicles 2018;6(22):29-37.

From: The Department of Internal Medicine, Texas Tech University Health Sciences Center, Lubbock, TX

Submitted: $11 / 20 / 2017$

Accepted: $1 / 6 / 2018$

Reviewer: Gilbert Berdine MD

Conflicts of interest: none

\section{REFERENCES}

1. Johnson KG. Treatment of sleep disordered breathing with positive airway pressure devices: technology update. Med Devices (Auckl) 2015 Oct 23;8:425-37.

2. Ambrogio C. Sleep and non-invasive ventilation in patients with chronic respiratory insufficiency. Intensive Care Med 2009 Feb;35(2):306-13.

3. Storre JH. Average volume-assured pressure support in obesity hypoventilation: A randomized crossover trial. Chest 2006 Sep;130(3):815-21.

4. Crisafulli E. Subjective sleep quality during average volume assured pressure support (AVAPS) ventilation in patients with hypercapnic COPD: A physiological pilot study. Lung 2009 Sep-Oct;187(5):299-305.

5. Murphy PB. Volume targeted versus pressure support non-invasive ventilation in patients with super obesity and chronic respiratory failure: a randomized controlled trial. Thorax 2012 Aug;67(8):727-34.

6. Briones Claudett KH, et al. Noninvasive mechanical ventilation with AVAPS in patients with COPD and hypercapnic encephalopathy. BMC Pulm Med 2013 Mar 12;13:12. doi: 10.1186/1471-2466-13-12.

7. Piesiak P. Efficacy of noninvasive volume targeted ventilation in patients with chronic respiratory failure due to kyphoscoliosis. Adv Exp Med Biol 2015;838:53-8. 